\title{
Nanoscale
}

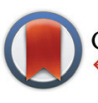

CrossMark

\&lick for updates

Cite this: Nanoscale, 2017, 9, 355

Received 10th August 2016,

Accepted 28th November 2016

DOI: $10.1039 / c 6 n r 06355 a$

www.rsc.org/nanoscale

\section{The single-molecule electrical conductance of a rotaxane-hexayne supramolecular assembly $\dagger$}

\author{
David C. Milan, $\$^{\mathrm{a}}$ Maximilian Krempe, $\$^{\mathrm{b}}$ Ali K. Ismael, $\ddagger^{\mathrm{c}, \mathrm{d}}$ Levon D. Movsisyan, $\xi^{\mathrm{e}}$ \\ Michael Franz, tb lain Grace, ${ }^{c}$ Richard J. Brooke, ${ }^{f}$ Walther Schwarzacher, ${ }^{f}$ \\ Simon J. Higgins, ${ }^{* a}$ Harry L. Anderson, ${ }^{*}$ Colin J. Lambert, ${ }^{* c}$ Rik R. Tykwinski*b and \\ Richard J. Nichols*a
}

\begin{abstract}
Oligoynes are archetypical molecular wires due to their 1-D chain of conjugated carbon atoms and ability to transmit charge over long distances by coherent tunneling. However, the stability of the oligoyne can be an issue. Here we address this problem by two stabilization methods, namely sterically shielding endgroups, and rotaxination to produce an insulated molecular wire. We demonstrate the threading of a hexayne within a macrocycle to form a rotaxane and report measurements of the electrical conductance of this single supramolecular assembly within an STM break junction. The macrocycle is retained around the hexayne through the use of 3,5-diphenylpyridine stoppers at both ends of the molecular wire, which also serve as chemisorption contacts to the gold electrodes of the junction. Molecular conductance was measured for both the supramolecular assembly and also for the molecular wire in the absence of the macrocycle. The threaded macrocycle, which at room temperature is mobile along the length of the hexayne between the stoppers, has only a minimal impact on the conductance. However, the probability of molecular junction formation in a given break junction formation cycle is notably lower with the rotaxane. In seeking to understand the conductance behavior, the electronic properties of these molecular assemblies and the electrical behavior of the junctions have been investigated by using DFT-based computational methods.
\end{abstract}

\section{Introduction}

In recent years, a variety of techniques has been introduced for reliably determining the conductance of single molecules. These techniques have employed scanning probe microscopes, ${ }^{1-4}$ break junctions ${ }^{5-7}$ or lithographic fabrication $^{8,9}$ to provide metal electrode pairs which can be controllably spaced to nanoscale dimensions and bridged by a small

\footnotetext{
${ }^{a}$ Department of Chemistry, University of Liverpool, Crown St, Liverpool, L69 7ZD, UK.E-mail: shiggins@liverpool.ac.uk,nichols@liv.ac.uk

${ }^{b}$ Department of Chemistry and Pharmacy \& Interdisciplinary Center for Molecular Materials (ICMM), University of Erlangen-Nürnberg (FAU), Henkestrasse 42, 91054 Erlangen, Germany. E-mail: rik.tykwinski@fau.de

${ }^{c}$ Department of Physics, University of Lancaster, Lancaster, LA1 4YB, UK. E-mail: c.lambert@lancaster.ac.uk

${ }^{d}$ Department of Physics, College of Education for Pure Science, Tikrit University, Tikrit, Iraq

${ }^{e}$ Department of Chemistry, University of Oxford, Chemistry Research Laboratory, Oxford, OX1 3TA, UK. E-mail: harry.anderson@chem.ox.ac.uk

${ }^{f}$ H. H. Wills Physics Laboratory, University of Bristol, Tyndall Avenue, Bristol BS8 1TL, UK

$\dagger$ Electronic supplementary information (ESI) available. See DOI: 10.1039/ c6nr06355a

$\$$ These authors contributed equally to this work.
}

number of molecules. They were initially used to study the conductance of small conjugated or saturated molecular bridges, for example benzene-1,4-dithiolate, 4,4'-bipyridine or alkanedithiols. ${ }^{3,6}$ More recently, they have been used to study molecules of increasing chemical and structural complexity and even bi-molecular and multi-molecular assemblies in junctions. Measurements often rely on non-covalent interactions such as hydrogen-bonding or van der Waals interactions to promote molecular assembly in the junction. Examples include assemblies formed from multiple DNA bases spanning the metallic contact gap, ${ }^{10}$ pairs of hydrogenbonding carboxylic acids, ${ }^{11}$ conjugated molecular bridges stacking through facing aromatic rings ${ }^{12,13}$ and fullerenes that assemble non-covalently with porphyrins within the electrical junction. ${ }^{14}$ Host-guest interactions have also been exploited, notably the threading of single molecular wires within cagelike molecular hosts such as $\alpha$-cyclodextrin ${ }^{15-17}$ or cucurbit[8] uril. $^{16-18}$ In these cases it was shown that encapsulation can suppress molecular aggregation ${ }^{15}$ or act to control the environment ${ }^{18}$ immediately surrounding the molecular wire.

Oligoynes represent a prototypical molecular bridge system for charge transport studies. ${ }^{19-25}$ They consist of alternating $\mathrm{C} \equiv \mathrm{C}$ and $\mathrm{C}-\mathrm{C}$ bonds in 1-D molecular carbon chains and they 
have been described as "model substances for the hypothetical one-dimensional carbon allotrope carbyne $\mathrm{C} \infty$ ". ${ }^{26}$ When appropriate anchoring groups are connected at either end of these chains they can be used to form single-molecule junctions using STM or break junction methods. Wang et al. first established the formation of oligoyne single-molecule electrical junctions in an STM with gold electrodes and pyridyl anchoring groups at both termini of the oligoyne chain. ${ }^{20}$ The conductance decay with length across a series of these oligomers was demonstrated to be low, in both experimental and theoretical studies. ${ }^{19,20}$ Further single-molecule experimental studies explored different anchoring groups at the chain termini and also the influence of the solvent medium on the molecular junction electrical properties. ${ }^{21,22}$

Oligoynes endcapped with pyridyl groups are viable candidates for single-molecular junction formation..$^{20-22,27}$ To date, however, the synthesis and study of oligoynes bearing unfunctionalized pyridyl groups has been limited to the length of a hexayne, ${ }^{22,28}$ due to the increasing instability of oligoynes with length. Recently, we disclosed the synthesis of a series of dipyridyloligoyne derivatives kinetically stabilized by the presence of 3,5-diphenyl substitution of the pyridine. ${ }^{29}$ In this case, steric shielding of the endmost ethynyl moieties led to significant stabilization compared to the unsubstituted series. ${ }^{29} \mathrm{~A}$ similar approach has been used to synthesize the longest known polyynes. ${ }^{30}$ Aside from sterically demanding endgroups, an alternative approach toward the stabilization of sphybridized carbon chains has been described based on rotaxane formation, for examples see ref. 31-37. It is easy to envisage the combination of both stabilization methods, namely sterically shielding endgroups and rotaxination, to produce an insulated molecular wire. Equally interesting is the possible effect of isolating the molecular wire from the electrical influences of neighboring molecules. ${ }^{38-42}$

Given the interest in electrically conducting oligoyne molecular wires, we explore here the possibility of forming singlemolecule junctions of an oligoyne threaded within a macrocycle. Rotaxane-based molecular assemblies have been considered as future candidates for molecular electronic devices, ${ }^{43}$ including concepts for nanoscale data recording. ${ }^{44}$ Singlemolecule electrical conductance is determined for the hexameric oligoyne rotaxane and also for the unthreaded hexayne. The impact of the threading on the conductance is analyzed and discussed with complementary transport computations using DFT.

\section{Experimental and theoretical methods}

\section{Conductance measurements}

An Agilent (Keysight) STM running Picoscan 5.3.3 software was used for all single-molecule conductance measurements, and these were performed at room temperature and under ambient conditions. Electrical measurements were performed by the STM-BJ technique using a home-made scanner ${ }^{45}$ controlled by a Agilent Picoscan 5500 Controller with a N9503A scanner which was modified with a custom multi-channel current amplifier. Molecular adlayers were formed by adsorption on $\mathrm{Au}(111)$ substrates. These commercially fabricated gold-onglass samples with a chromium adhesion layer (Arrandee) were prepared by flame-annealing immediately prior to use. The flame-annealing procedure involved heating the gold slide until it developed a slight orange colour as viewed under darkened room conditions. The slide was kept in this state for approximately $30 \mathrm{~s}$ by moving it in and out of the flame. Care was taken to ensure that the gold slide did not overheat. The flame-heating was performed three times to generate flat $\mathrm{Au}(111)$ terraces through annealing. ${ }^{46}$ Sample solutions were prepared by adding $1 \mathrm{~mL}$ of chloroform to a vial with $0.2 \mathrm{mg}$ of the relevant compound. Molecular adsorption was achieved by immersing the gold slide in the chloroform solutions of the compound for 20 minutes. The sample was then rinsed with ethanol and dried under a moderate flow of gaseous nitrogen. Gold STM tips were fabricated from $0.25 \mathrm{~mm}$ Au wire (99.99\%) that was freshly mechanically cut for each STM experiment.

For conductance determination the STM-BJ tip was repeatedly brought into contact and then withdrawn from the surface. An STM set point current of $100 \mu \mathrm{A}$ was used to drive the gold STM tip into the surface, followed by retracting the tip $4 \mathrm{~nm}$ away from the surface at a rate of $20 \mathrm{~nm} \mathrm{~s}^{-1}$. A small bias voltage of $-300 \mathrm{mV}$ was applied between the tip and substrate and the current versus retraction distance traces were recorded. If no molecules became bound between the tip and the surface while withdrawing the tip, a smooth exponential decay was observed in the current trace. However, when one or more molecules bridged between the tip and the surface, steps were observed. The cycle of driving the tip into the surface and retraction was repeated $\sim 4000$ times for each sample, after which the current versus distance traces were analyzed. An automated procedure was employed to create conductance histograms from the raw current-distance data, which algorithmically rejects some curves with excessive noise or extremely long decay times. ${ }^{45}$ The peaks obtained in the histograms at $G \ll G_{0}$ (where $G_{0}$ is the metallic point contact conductance) represent the most likely conductance values for molecular junctions.

\section{Computational details}

Electronic structure calculations were performed using the DFT code SIESTA. ${ }^{47}$ The optimum geometry of the isolated rotaxane and dumbbell molecules were obtained by relaxing the molecules until all forces on the atoms were less than 0.05 eV $\AA^{-1}$. The SIESTA calculations employed a double-zeta plus polarization orbital basis set, norm-conserving pseudopotentials, an energy cutoff of 200 Rydergs defined the real space grid and the exchange correlation functional was LDA. To calculate the conductance through these two molecules, they were attached to gold leads via the pyridyl anchor groups. The leads were constructed of 6 layers of $\mathrm{Au}(111)$ each containing 30 gold atoms and the optimum binding distance was calculated to be $2.4 \AA$ (see Fig. $\mathrm{S} 1 \dagger$ ) between the terminal nitrogen atoms and a 'top' gold atom. A Hamiltonian describing this structure was produced using SIESTA and the zero-bias trans- 
mission coefficient $T(E)$ was calculated using the Gollum code. Further details are provided in the ESI. $\dagger$

\section{Synthesis and characterization}

Details and spectra are provided in the ESI. $\dagger$

\section{Results and discussion}

The two compounds studied in the present work are a dumbbell-shaped oligoyne with two bulky "stoppers" (compound 3, referred to as the "dumbbell"), and the same dumbbell molecule that is threaded with a phenanthroline containing macrocycle (M1), referred to here as the "rotaxane" (3·M1, see Scheme 1). Macrocycle M1 has been previously utilised successfully in oligoyne and cumulene rotaxanes synthesis. ${ }^{31,32}$ The small size, the rigid structure and synthetic feasibility makes macrocycle $\mathbf{1}$ a suitable thread for pyridine-capped dumbbell-shaped oligoynes. The synthesis of rotaxane 3·M1 began with the formation of triynes $\mathbf{1 a}$ and $\mathbf{1 b}$ (Scheme 1) as previous reported. ${ }^{29}$ Treatment of $\mathbf{1 a}$ with TBAF in wet THF furnished terminal alkyne 2 , which was not isolated due to its tendency to rapidly decompose over the course of seconds to minutes. Rather, the desilylated intermediate 2 was carried on, following work up, to rotaxane formation.

The estimated maximum diameter of the 3,5-diphenylpyridyl endcapping group is $c a$. $10 \AA^{29},{ }^{29}$ and thus the known phenanthroline-based macrocycle $\mathbf{M 1},{ }^{31}$ which features a cavity size of ca. $8.5 \AA$, was chosen as second component for the rotaxane (Scheme 1). The diameters of the 3,5-diphenylpyridyl endcapping group and M1 were estimated from the X-ray crystallographic analyses reported in ref. 29 and 31 respectively. The phenanthroline-Cu(I) complex M1·Cu(I) was formed via the reaction of macrocycle $\mathbf{M 1}$ with $\mathrm{CuI}$ in $\mathrm{CH}_{2} \mathrm{Cl}_{2} / \mathrm{CH}_{3} \mathrm{CN}$.

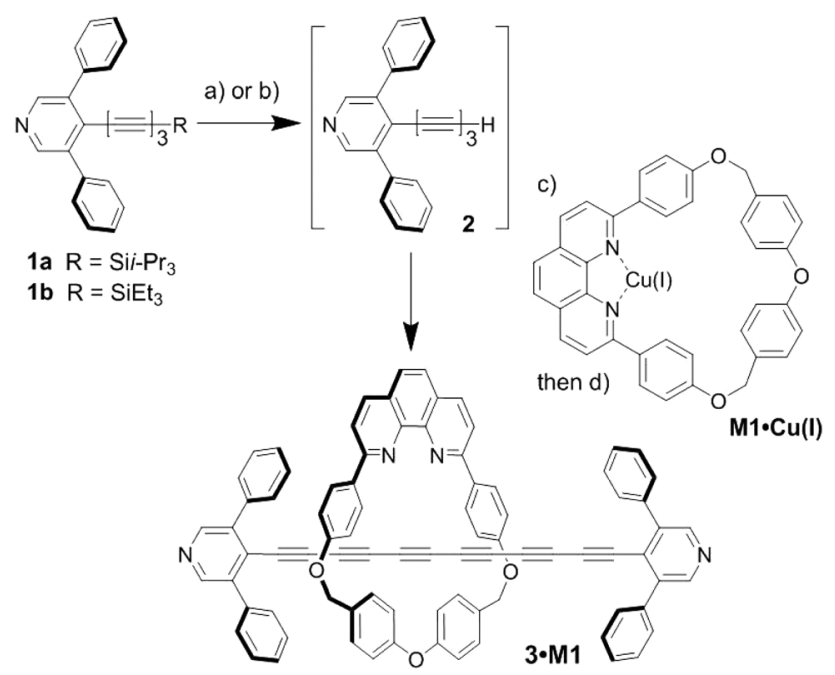

Scheme 1 Hexayne rotaxane synthesis. Reagents and conditions: (a) with $\mathrm{R}=\mathrm{Sii}-\mathrm{Pr}_{3}$, TBAF, wet THF, rt. (b) With $\mathrm{R}=\mathrm{SiEt}_{3}, \mathrm{CsF}$, wet THF, rt. (c) M1. Cu(I), $\mathrm{K}_{2} \mathrm{CO}_{3}, \mathrm{I}_{2}, \mathrm{THF}, 60^{\circ} \mathrm{C}$. (d) $\mathrm{KCN}$ (in $\mathrm{H}_{2} \mathrm{O}$ ), $\mathrm{CH}_{2} \mathrm{Cl}_{2}, \mathrm{CH}_{3} \mathrm{CN}$, rt ( $2 \%$ yield over three steps from 1a; $13 \%$ yield over three steps from $1 \mathrm{~b}$ ).
Triyne $\mathbf{2}$ in THF was then added to the M1.Cu(I) complex, and the mixture was stirred under nitrogen at $60^{\circ} \mathrm{C}$ in the presence of $\mathrm{K}_{2} \mathrm{CO}_{3}$ and $\mathrm{I}_{2}$ until the reaction was judged complete (24 h, as monitored by TLC). Cu(I) was removed with aqueous KCN, and rotaxane $\mathbf{3 \cdot \mathbf { M } 1}$ was isolated in $2 \%$ yield via column chromatography (over the three steps from 1a).

Although easier to synthesize and manipulate, i-Pr $\mathrm{Si}_{3}$-protected triyne 1a required desilylation with TBAF, which can lead to loss of material via decomposition. Thus, $\mathrm{Et}_{3} \mathrm{Si}$-protected $\mathbf{1 b}$ was explored as an alternative, which can be desilylated under milder conditions with CsF. Using $\mathbf{1 b}$, and an analogous protocol as described for $\mathbf{1 a}$, the yield of rotaxane $\mathbf{3 \cdot \mathbf { M 1 }}$ $(13 \%)$ was indeed increased. As a model for comparison, the corresponding dumbbell 3 was synthesized as previously reported. $^{29}$

Characterization by ${ }^{1} \mathrm{H}$ and ${ }^{13} \mathrm{C}$ NMR spectroscopy shows that the spectra of 3.M1 are essentially linear combinations of the spectra of the macrocyclic and polyyne components M1 and 3, respectively. No significant changes in the spectra of 3.M1 are observed. Previous studies of the mobility of phenanthroline-based macrocycles threaded around oligoynes $(n \geq 2)$ showed that macrocycle is mobile even at $200 \mathrm{~K}$, and its rotation is faster than the NMR timescale $(0.2 \mathrm{~ms}) .{ }^{48}$ Moreover, the macrocycle was found to be highly mobile in a hexayne [3] rotaxane where there is a significant restriction due to steric hindrance. $^{32}$

Rotaxane 3.M1 was analyzed by differential scanning calorimetry (DSC) and showed a melting endotherm at $124{ }^{\circ} \mathrm{C}$, followed immediately by decomposition of the rotaxane in the liquid phase. This behavior is similar to other reported hexayne rotaxanes, in which rotaxination leads to melting, rather than direct decomposition as often observed for the corresponding "naked" hexayne. ${ }^{32}$ For comparison, dumbbell 3 decomposes at $178{ }^{\circ} \mathrm{C}$, without melting (see ref. 29 for details).

The lowest energy UV-vis absorption of $3 \cdot \mathbf{M} 1\left(\lambda_{\max }=\right.$ $478 \mathrm{~nm}$ ) shows a slight redshift in comparison to the polyyne $3\left(\lambda_{\max }=474 \mathrm{~nm}\right)$. In the high energy region $(280-380 \mathrm{~nm})$, the absorption energies of the $\mathbf{3 \cdot M 1}$ are unchanged relative to $\mathbf{3}$, although the relative absorption intensities change, due to absorptions by the macrocycle. No significant solvatochromism was observed for 3·M1, over a range of polar and apolar solvents (see ESI $\dagger$ for spectra). Interestingly, measurements in $\mathrm{CHCl}_{3}$ "out of the bottle" revealed that 3.M1 was readily protonated by residual acidic impurities, and the protonated rotaxane shows a red-shifted $\lambda_{\max }$ value $\left(\lambda_{\max }=488 \mathrm{~nm}\right.$, see $\left.\operatorname{ESI} \dagger\right)$. Protonation could be effected completely by trifluoroacetic acid, and the process was reversible via addition of triethylamine (see ESI $\dagger$ ). An analogous redshift of $\lambda_{\max }$ is observed in the UV-vis spectrum of the protonated polyyne 3 . Thus, the changes in the electronic absorption of $\mathbf{3 \cdot M 1}$ are ascribed to protonation of the pyridyl groups (rather than the phenanthroline moiety within the macrocycle M1) and result from stabilization of the LUMO by protonation. ${ }^{49-52}$

Electrical conductance has been investigated here with the scanning tunneling microscope break junction technique 

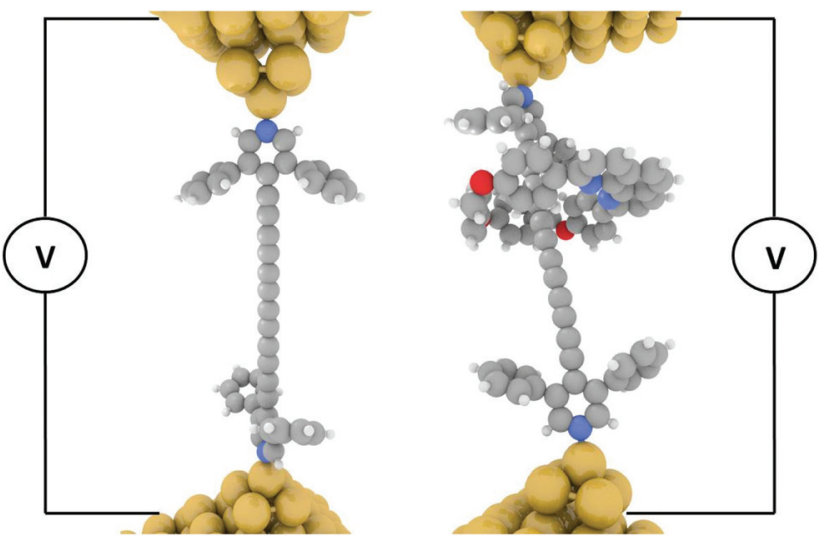

Fig. 1 Schematic of the STM-BJ molecular junction for single molecular conductance measurements. Left dumbbell (3), right rotaxane (3.M1).
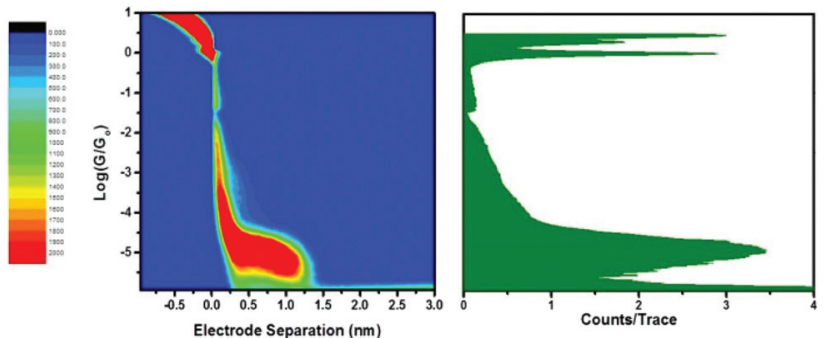

Fig. 2 Left: 2-D histogram showing the probability of a conductance $G$ at an electrode separation $z$, constructed from 3773 measured break junction traces for 3 . Right: The same data plotted as a 1-D conductance histogram.

(STM-BJ). This technique facilitates the repeated formation of molecular junctions and the determination of molecular conductance at the single-molecule level with large statistical datasets. $^{3}$ The STM-BJ method and related STM techniques have been widely used to study conductive $\pi$-conjugated molecular wires such as oligo(phenylene ethynylenes), oligo(phenylene vinylenes) and oligoynes. ${ }^{53-55}$

STM-BJ measurements were carried out for 3, 3·M1 (Fig. 1), and as a control, bare gold slides. Fig. 2 shows 1- and 2-dimensional histograms for the dumbbell. The 1-D histogram shows a sharp $1 G_{0}$ peak corresponding to the conductance of an Au point contact. Clear molecular conductance peaks can be seen at conductance values around $10^{-5} G_{0}$ in both the 1- and 2-D histograms. In the 2-D histogram, a long conductance plateau is observed with a plateau length of around $1 \mathrm{~nm}$. This could correspond to a rather invariant molecular conductance as the molecule is either pulled up in the junction from a tilted to upright orientation or as the molecule slides along the respective gold contacts until the molecular junction snaps. Fig. 3 compares the normalized 1-D histograms for 3 and a bare gold control sample, clearly showing the molecular conductance peak for 3 .

The rotaxane-hexayne supramolecular assembly (3·M1) was measured using the same conditions, giving the histograms

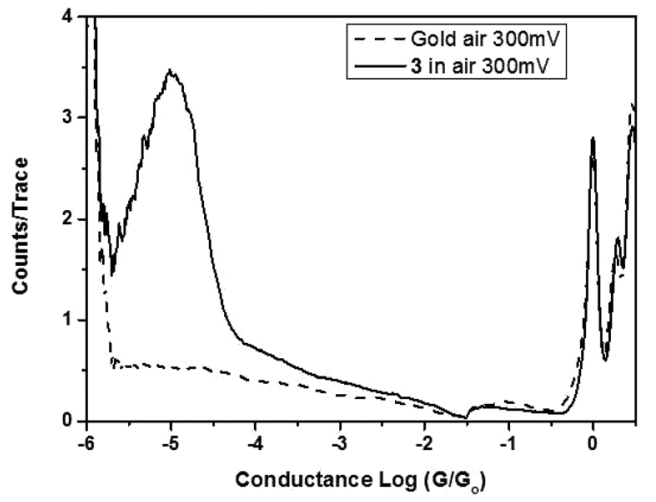

Fig. 3 1-D conductance histograms for the dumbbell 3 constructed from 3773 measured traces and for the blank gold sample constructed from 2010 traces. The histograms have been normalized by dividing the number of counts by the numbers of scans.
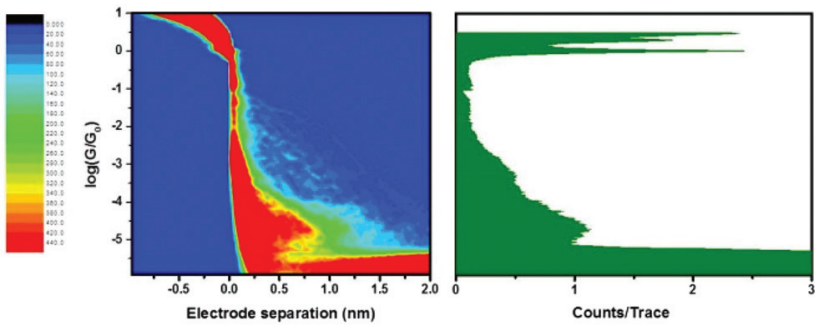

Fig. 4 Left: 2-D histogram showing the probability of a conductance $G$ at an electrode separation $z$, constructed from 3472 measured break junction traces for 3.M1. Right: The same data plotted as a 1-D conductance histogram.

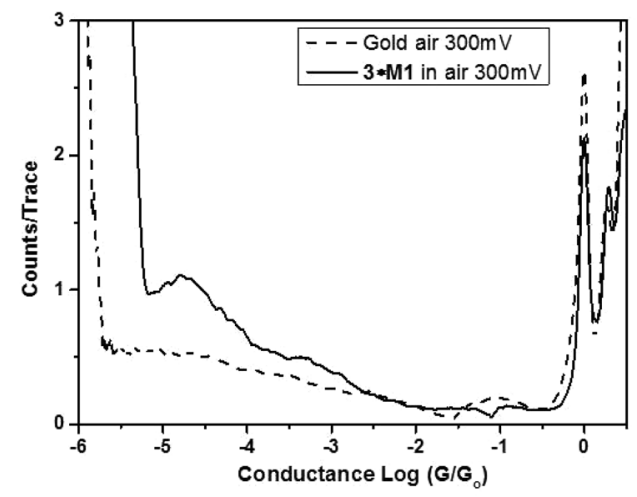

Fig. 5 Conductance histograms for the rotaxane 3.M1 constructed from 3472 measured traces for 3.M1 and 2010 traces for the gold. Histograms have been normalized by dividing the number of events by the numbers of scans.

shown in Fig. 4. This figure shows a molecular conductance peak at slightly greater than $10^{-5} G_{0}$ indicating that rotaxane formation only influences the conductance of the hexayne thread to a relatively small extent (Fig. 5); note however that the length of the conductance plateau in the 2D histogram in 


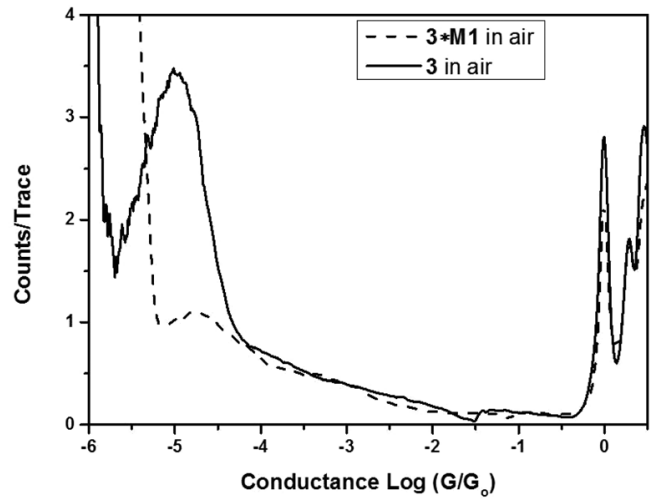

Fig. 6 Comparison of the 1-D conductance histograms for 3.M1 and 3.

Table 1 Conductance values for the molecular targets

\begin{tabular}{ll}
\hline & Conductance \\
\hline 3 & $(0.97 \pm 0.17) \times 10^{-5} G_{0}$ \\
$3 \cdot \mathbf{M 1}$ & $(1.66 \pm 0.25) \times 10^{-5} G_{0}$
\end{tabular}

Fig. 4 is considerably shorter than the corresponding feature in Fig. 2. The two histograms are plotted together in Fig. 6 and the conductance peak values are summarized in Table 1 . The conductance values here are broadly consistent with previous results, ${ }^{20-22}$ although an absolute comparison should be made with caution since different surface anchoring groups and different media are employed. For a pyridine capped oligoyne with 4 triple bonds conductance values in the range of $2 \times$ $10^{-3} G_{0}$ to $5 \times 10^{-5} G_{0}$, depending on the conductance group, have been reported. ${ }^{20}$ In another study significant dependence on solvent medium has been shown. ${ }^{22}$ Ref. 21 showed that upon elongation of carbon chain from 1 to 4 triple bonds, also in the series of pyridine tethered oligoynes, the high conductance values obtained by STM-BJ varied from $4.0 \times 10^{-4} G_{0}$ (for $n=1)$ to $4.0 \times 10^{-5} G_{0}$ (for $\left.n=4\right)$. By comparison, the conductance of the hexayne molecule in our experiments is $(0.97 \pm$ $0.17) \times 10^{-5} G_{0}$ (Table 1).

From Table 1 it can be seen that the threaded macrocycle appears to increase the conductance of the hexayne molecular wire, but any effect is rather small. Since the $y$-scale is normalized in counts per trace, the lower peak for the 3.M1 indicates a lower probability for junction formation. This can be interpreted as the bulky phenanthroline-containing macrocycle positioned between the stoppers of the hexayne molecular wire inhibiting molecular bridge formation as illustrated schematically in Fig. 7. This model shows junction formation for 3 occurring by the compound sliding or diffusing along the contacts to attach to the respective electrodes through binding between the terminal pyridyl group and the gold contacts. Such a mechanism might be impeded with the bulky phenanthroline containing macrocycle in place in $\mathbf{3 \cdot M 1}$, which is also consistent with the shorter plateau length observed for 3·M1

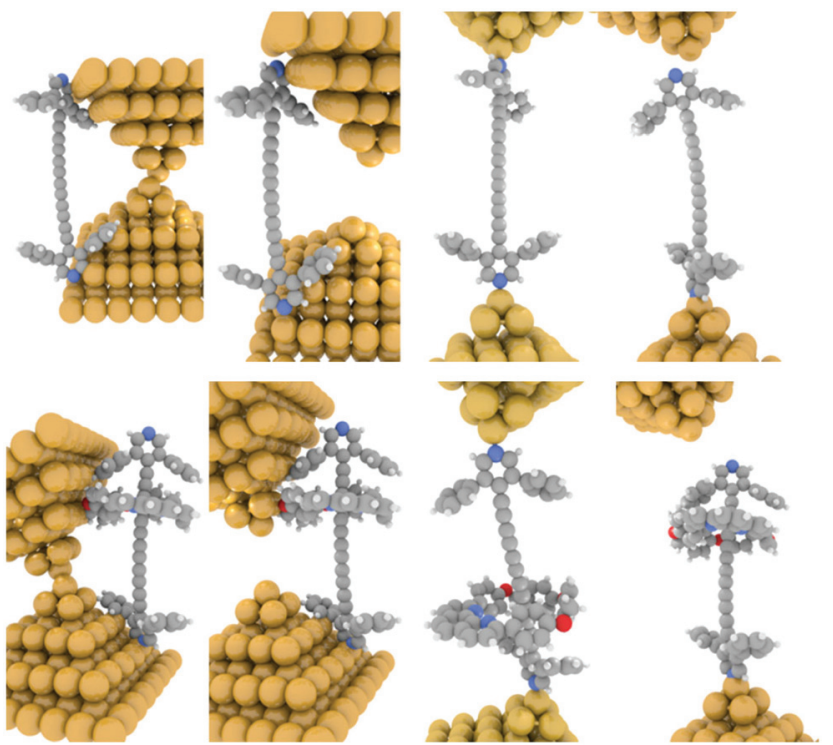

Fig. 7 Schematic illustration of a possible sequence of events leading to junction formation with 3 (top sequence) and 3.M1 (bottom sequence), respectively.

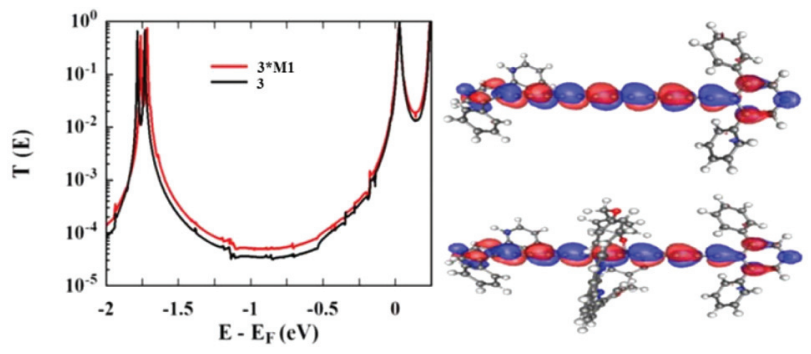

Fig. 8 Left: Zero bias transmission coefficient curves, $T(E)$ of 3 and 3.M1. Right: LUMO orbital of 3 (top) and 3.M1 (bottom).

than 3. Although the phenanthroline macrocycle also contains pyridyl $\mathrm{N}$ atoms, their interaction with gold is expected to be extremely weak compared to the interaction with pyridine endgroups, due to the spatial geometry and steric hindrance.

DFT computations have been performed to understand further the influence on the molecular conductance of threading the hexayne through the phenanthroline containing macrocycle. Fig. 8 shows the transmission coefficient for $\mathbf{3} \cdot \mathbf{M} 1$ (red) and 3 (black), which are virtually identical, in agreement with the experimental measurements which show both molecules having a broadly similar conductance value of $\sim 10^{-5} G_{0}$. Both curves show LUMO dominated transport with the DFT calculated Fermi energy $(0 \mathrm{eV})$ sitting close to LUMO resonance and 3.M1 showing a slightly higher conductance value in the gap between the HOMO and LUMO. Examination of the orbitals of the isolated molecules can explain why the macrocycle of the rotaxane plays no part in the charge transport. Fig. 8 shows that the LUMO orbital of each molecule is identical. The LUMO of 3.M1 shows no weight on the ring and there- 
fore plays no role in conductance. Further analysis of the frontier orbitals show that the HOMO of 3.M1 is localized on the ring (Fig. S5 $\mathbf{H}^{\dagger}$ ) and it is the HOMO-5 orbital of $\mathbf{3 \cdot \mathbf { M } 1}$ which is delocalized along the oligoyne. The HOMO-5 of 3.M1 and the HOMO of 3 are identical.

\section{Conclusions}

Oligoynes previously-studied in single molecule conductance measurements typically had $\leq 5$ triple bonds, including the family of 4-pyridyl-contacted oligoynes studied by ourselves and others; longer examples would be unstable under the conditions employed. In contrast, in the present work we show that the employment of 3,5-diphenyl substitution that is known to make the pyridyl terminal (contact) group more protective of the oligoyne moiety indeed allows us to measure the conductance of an oligoyne with 6 triple bonds. We also show that although it is known from our previous work that the rotaxane provides mechanical protection of the oligoyne chain, ${ }^{32}$ in single molecule junction measurements the rotaxane shows smaller propensity for single molecule junction formation. These conclusions are reached by determining the single-molecular conductance of a dumbbell shaped hexayne molecular wire (3) and the same dumbbell compound threaded into a phenanthroline containing macrocycle (3·M1) by the STM-BJ technique and DFT transport computations. A slight increase of the conductance for the rotaxane (3.M1) was seen compared with the dumbbell (3). However, threading the stoppered molecular wire into a macrocycle resulted in a marked decrease in the propensity of the target to form molecular junctions. Computational data agree with the experimentally observed small increase of the conductance for the rotaxane molecule, with the increase being small since the phenanthroline macrocycle has little effect on either the orbital energetics or orbital densities of the charge transporting frontier molecular orbitals of the molecular wire. In this respect, the macrocycle hosting $\mathbf{3}$ is a good choice if one wishes to study long range charge transport through stable oligoyne molecular wires with little "outside" electronic perturbation. However, as a perspective, other choices of macrocycle showing a stronger electronic interaction with the oligoyne chain, may be employed to modulate or gate the conductance of the molecular wire.

\section{Acknowledgements}

RJN and SJH thank EPSRC for funding (EP/K007785/1 and EP/ M014169/1). RRT, MF, and MK are grateful for funding from the University of Erlangen-Nürnberg, the Deutsche Forschungsgemeinschaft (DFG - SFB 953, "Synthetic Carbon Allotropes" and the DFG grant "Rotaxane Protected Polyynes and Cumulenes"). LDM acknowledges an Oxford University Raffy Manoukian scholarship. AKI acknowledges financial support from Tikrit University.

\section{References}

1 X. D. Cui, A. Primak, X. Zarate, J. Tomfohr, O. F. Sankey, A. L. Moore, T. A. Moore, D. Gust, G. Harris and S. M. Lindsay, Science, 2001, 294, 571-574.

2 W. Haiss, H. van Zalinge, S. J. Higgins, D. Bethell, H. Hobenreich, D. J. Schiffrin and R. J. Nichols, J. Am. Chem. Soc., 2003, 125, 15294-15295.

3 B. Q. Xu and N. J. J. Tao, Science, 2003, 301, 1221-1223.

4 W. Haiss, H. van Zalinge, H. Hobenreich, D. Bethell, D. J. Schiffrin, S. J. Higgins and R. J. Nichols, Langmuir, 2004, 20, 7694-7702.

5 C. Kergueris, J. P. Bourgoin, S. Palacin, D. Esteve, C. Urbina, M. Magoga and C. Joachim, Phys. Rev. B: Condens. Matter, 1999, 59, 12505-12513.

6 M. A. Reed, C. Zhou, C. J. Muller, T. P. Burgin and J. M. Tour, Science, 1997, 278, 252-254.

7 H. B. Weber, J. Reichert, F. Weigend, R. Ochs, D. Beckmann, M. Mayor, R. Ahlrichs and $H$. von Lohneysen, Chem. Phys., 2002, 281, 113-125.

8 S. Kubatkin, A. Danilov, M. Hjort, J. Cornil, J. L. Bredas, N. Stuhr-Hansen, P. Hedegard and T. Bjornholm, Nature, 2003, 425, 698-701.

9 E. A. Osorio, K. O’Neill, N. Stuhr-Hansen, O. F. Nielsen, T. Bjornholm and H. S. J. van der Zant, Adv. Mater., 2007, 19, 281-285.

10 S. Huang, J. He, S. Chang, P. Zhang, F. Liang, S. Li, M. Tuchband, A. Fuhrmann, R. Ros and S. Lindsay, Nat. Nanotechnol., 2010, 5, 868-873.

11 T. Nishino, N. Hayashi and P. T. Bui, J. Am. Chem. Soc., 2013, 135, 4592-4595.

12 S. Martin, I. Grace, M. R. Bryce, C. Wang, R. Jitchati, A. S. Batsanov, S. J. Higgins, C. J. Lambert and R. J. Nichols, J. Am. Chem. Soc., 2010, 132, 91579164.

13 S. M. Wu, M. T. Gonzalez, R. Huber, S. Grunder, M. Mayor, C. Schonenberger and M. Calame, Nat. Nanotechnol., 2008, 3, 569-574.

14 P. T. Bui, T. Nishino, Y. Yamamoto and H. Shiigi, J. Am. Chem. Soc., 2013, 135, 5238-5241.

15 M. Kiguchi, S. Nakashima, T. Tada, S. Watanabe, S. Tsuda, Y. Tsuji and J. Terao, Small, 2012, 8, 726-730.

16 F. H. Huang and H. W. Gibson, Prog. Polym. Sci., 2005, 30, 982-1018.

17 M. Xue, Y. Yang, X. D. Chi, X. Z. Yan and F. H. Huang, Chem. Rev., 2015, 115, 7398-7501.

18 W. Zhang, S. Gan, A. Vezzoli, R. J. Davidson, D. C. Milan, K. V. Luzyanin, S. J. Higgins, R. J. Nichols, A. Beeby, P. J. Low, B. Li and L. Niu, ACS Nano, 2016, 5212-5220.

19 Z. Crljen and G. Baranovic, Phys. Rev. Lett., 2007, 98, 116801.

20 C. Wang, A. S. Batsanov, M. R. Bryce, S. Martin, R. J. Nichols, S. J. Higgins, V. M. Garcia-Suarez and C. J. Lambert, J. Am. Chem. Soc., 2009, 131, 1564715654. 
21 P. Moreno-Garcia, M. Gulcur, D. Z. Manrique, T. Pope, W. Hong, V. Kaliginedi, C. Huang, A. S. Batsanov, M. R. Bryce, C. Lambert and T. Wandlowski, J. Am. Chem. Soc., 2013, 135, 12228-12240.

22 D. C. Milan, O. A. Al-Owaedi, M.-C. Oerthel, S. MarquésGonzález, R. J. Brooke, M. R. Bryce, P. Cea, J. Ferrer, S. J. Higgins, C. J. Lambert, P. J. Low, D. Z. Manrique, S. Martin, R. J. Nichols, W. Schwarzacher and V. M. GarcíaSuárez, J. Phys. Chem. C, 2016, 120, 15666-15674.

23 V. M. Garcia-Suarez and C. J. Lambert, Nanotechnology, 2008, 19, 455203.

24 C. J. Lambert, Chem. Soc. Rev., 2015, 44, 875-888.

25 A. Al-Backri, V. Zolyomi and C. J. Lambert, J. Chem. Phys., 2014, 140.

26 T. Gibtner, F. Hampel, J. P. Gisselbrecht and A. Hirsch, Chem. - Eur. J., 2002, 8, 408-432.

27 M. Gulcur, P. Moreno-Garcia, X. Zhao, M. Baghernejad, A. S. Batsanov, W. Hong, M. R. Bryce and T. Wandlowski, Chem. - Eur. J., 2014, 20, 4653-4660.

28 A recent report of a hexayne endcapped with pyridyl groups has appeared (in Chinese), although this compound is unstable to isolation, see. C. Wang, H. Jia, T. Li and Y. Wang, Jilin Huagong Xueyuan Xuebao, 2012, 29, 33.

29 M. Krempe, R. Lippert, F. Hampel, I. Ivanovic-Burmazovic, N. Jux and R. R. Tykwinski, Angew. Chem., Int. Ed., 2016, 55, 14802-14806.

30 W. A. Chalifoux and R. R. Tykwinski, Nat. Chem., 2010, 2, 967-971.

31 M. Franz, J. A. Januszewski, D. Wendinger, C. Neiss, L. D. Movsisyan, F. Hampel, H. L. Anderson, A. Goerling and R. R. Tykwinski, Angew. Chem., Int. Ed., 2015, 54, 66456649.

32 L. D. Movsisyan, M. Franz, F. Hampel, A. L. Thompson, R. R. Tykwinski and H. L. Anderson, J. Am. Chem. Soc., 2016, 138, 1366-1376.

33 L. D. Movsisyan, D. V. Kondratuk, M. Franz, A. L. Thompson, R. R. Tykwinski and H. L. Anderson, Org. Lett., 2012, 14, 3424-3426.

34 S. Saito, E. Takahashi and K. Nakazono, Org. Lett., 2006, 8, 5133-5136.

35 S. Saito, E. Takahashi, K. Wakatsuki, K. Inoue, T. Orikasa, K. Sakai, R. Yamasaki, Y. Mutoh and T. Kasama, J. Org. Chem., 2013, 78, 3553-3560.

36 N. Weisbach, Z. Baranova, S. Gauthier, J. H. Reibenspies and J. A. Gladysz, Chem. Commun., 2012, 48, 75627564 .
37 S. Schrettl, E. Contal, T. N. Hoheisel, M. Fritzsche, S. Balog, R. Szilluweit and H. Frauenrath, Chem. Sci., 2015, 6, 564574 .

38 S. Anderson, R. T. Aplin, T. D. W. Claridge, T. Goodson, A. C. Maciel, G. Rumbles, J. F. Ryan and H. L. Anderson, J. Chem. Soc., Perkin Trans. 1, 1998, 2383-2397, DOI: 10.1039/a802680g.

39 F. Cacialli, J. S. Wilson, J. J. Michels, C. Daniel, C. Silva, R. H. Friend, N. Severin, P. Samori, J. P. Rabe, M. J. O'Connell, P. N. Taylor and H. L. Anderson, Nat. Mater., 2002, 1, 160-164.

40 M. J. Frampton and H. L. Anderson, Angew. Chem., Int. Ed., 2007, 46, 1028-1064.

41 J. Terao, Polym. Chem., 2011, 2, 2444-2452.

42 J. Terao, K. Homma, Y. Konoshima, R. Imoto, H. Masai, W. Matsuda, S. Seki, T. Fujihara and Y. Tsuji, Chem. Commun., 2014, 50, 658-660.

43 C. Joachim, J. K. Gimzewski and A. Aviram, Nature, 2000, 408, 541-548.

44 M. Cavallini, F. Biscarini, S. Leon, F. Zerbetto, G. Bottari and D. A. Leigh, Science, 2003, 299, 531-531.

45 R. J. Brooke, C. Jin, D. S. Szumski, R. J. Nichols, B.-W. Mao, K. S. Thygesen and W. Schwarzacher, Nano Lett., 2015, 15, 275-280.

46 W. Haiss, D. Lackey, J. K. Sass and K. H. Besocke, J. Chem. Phys., 1991, 95, 2193-2196.

47 C. M. Finch, V. M. Garcia-Suarez and C. J. Lambert, Phys. Rev. B: Condens. Matter, 2009, 79, 033405.

48 M. J. Langton, J. D. Matichak, A. L. Thompson and H. L. Anderson, Chem. Sci., 2011, 2, 1897-1901.

49 F. Bures, D. Cvejn, K. Melanova, L. Benes, J. Svoboda, V. Zima, O. Pytela, T. Mikysek, Z. Ruzickova, I. V. Kityk, A. Wojciechowski and N. AlZayed, J. Mater. Chem. C, 2016, 4, 468-478.

50 G. Cauzzo, G. Galiazzo, U. Mazzucat and N. Mongiat, Tetrahedron, 1966, 22, 589-593.

51 B. Hu, X. Chen, Y. Wang, P. Lu and Y. Wang, Chem. - Asian J., 2013, 8, 1144-1151.

52 U. Mazzucato and A. Spalletti, Photochem. Photobiol. Sci., 2003, 2, 282-288.

53 D. Xiang, X. Wang, C. Jia, T. Lee and X. Guo, Chem. Rev., 2016, 116, 4318-4440.

54 N. J. Tao, Nat. Nanotechnol., 2006, 1, 173-181.

55 R. J. Nichols, W. Haiss, S. J. Higgins, E. Leary, S. Martin and D. Bethell, Phys. Chem. Chem. Phys., 2010, 12, 28012815. 